\title{
La autonomía económica de las mujeres en tiempos de COVID-19
}

\author{
Nicole Bidegain, Lucía Scuro e Iliana Vaca Trigo
}

\section{Resumen}

Este artículo contribuye al debate sobre los efectos de la crisis causada por la pandemia de enfermedad por coronavirus (COVID-19) en las desigualdades de género en América Latina y el Caribe, con foco en la autonomía económica de las mujeres. Mediante una revisión de los aportes de la economía feminista y un análisis de la evidencia empírica se constata un deterioro de los indicadores asociados al vínculo de las mujeres con el mercado laboral y los ingresos. La división sexual del trabajo se está profundizando en la región y pone en riesgo los escasos avances en términos de autonomía económica de las mujeres alcanzados en los años previos a la crisis. Se alerta sobre los sesgos de género de las políticas de mitigación de la crisis y se reflexiona sobre la importancia de redistribuir el tiempo, los recursos y el poder para transitar hacia un nuevo estilo de desarrollo basado en la igualdad de género y la sostenibilidad.

\section{Palabras clave}

COVID-19, virus, epidemias, aspectos económicos, feminismo, mujeres, ingresos, mercado de trabajo, empleo de la mujer, tiempo, igualdad de género, América Latina y el Caribe

\section{Clasificación JEL}

B54, 054, D31, 138 y J22

\section{Autoras}

Nicole Bidegain es Oficial de Asuntos Sociales de la División de Asuntos de Género de la Comisión Económica para América Latina y el Caribe (CEPAL). Correo electrónico: nicole.bidegain@cepal.org.

Lucía Scuro es Oficial de Asuntos Sociales de la División de Asuntos de Género de la Comisión Económica para América Latina y el Caribe (CEPAL). Correo electrónico: lucia.scuro@cepal.org.

lliana Vaca Trigo es Estadística de la División de Asuntos de Género de la Comisión Económica para América Latina y el Caribe (CEPAL). Correo electrónico: iliana.vacatrigo@cepal.org. 


\section{Introducción}

La pandemia de COVID-19 en América Latina y el Caribe ha desencadenado múltiples crisis con efectos distributivos diferentes para hombres y mujeres. En esta coyuntura se ha puesto de manifiesto el carácter estructural de las desigualdades de género en la región y la mayor exposición de las mujeres a los efectos de las crisis. Esta pandemia irrumpe en un contexto marcado por asimetrías en el plano global, bajo crecimiento de las economías de la región, deterioro de la calidad del empleo y crecientes desigualdades en países con sistemas de salud fragmentados, mercados laborales segmentados y políticas de igualdad de género con recursos insuficientes. Como en crisis anteriores, las mujeres parecen amortiguar los efectos mediante una intensificación del trabajo doméstico y de cuidados no remunerado (CEPAL, 2020d y 2020f).

En la literatura reciente existe consenso sobre los efectos de la crisis en las mujeres, tanto en países desarrollados como en países y regiones en desarrollo (Naciones Unidas, 2020). En diferentes trabajos se abordan la profundidad de los efectos, los factores explicativos de dicho fenómeno, las dimensiones de la desigualdad de género que se analizan y su intersección con otros factores como los ingresos, la edad y la raza-etnia, así como su vínculo con las estructuras productivas.

El objetivo de este artículo es contribuir al debate sobre los efectos de la crisis del COVID-19 en las desigualdades de género en América Latina y el Caribe, con foco en la autonomía económica de las mujeres, y determinar los principales factores estructurales subyacentes que operan y que deben subvertirse para evitar la profundización de las desigualdades de género a mediano y largo plazo. En la sección Il se hace una revisión de la literatura sobre los principales aportes de la economía feminista para comprender los vínculos entre la dinámica económica y las desigualdades de género, en particular en contextos de crisis. En la sección subsiguiente se analizan algunos indicadores de autonomía económica de las mujeres sobre la base de evidencia empírica disponible de fuentes oficiales y proyecciones de la Comisión Económica para América Latina y el Caribe (CEPAL). En la sección IV se presentan algunas reflexiones sobre el enfoque de las medidas de mitigación y reactivación para avanzar hacia un nuevo estilo de desarrollo basado en la igualdad de género y la sostenibilidad.

\section{Crisis, teoría feminista y COVID-19 en América Latina y el Caribe}

En el marco de la economía feminista, como corriente de pensamiento heterodoxo, se han desarrollado herramientas conceptuales y metodológicas para comprender la forma en que las relaciones de género permean el sistema económico y sus dinámicas. Estas herramientas son de utilidad para abordar las externalidades de la crisis actual en la vida de las mujeres y proponer transformaciones profundas en el estilo de desarrollo dominante. A diferencia de la economía de género, la economía feminista se presenta como pensamiento transformador, al proponer un nuevo paradigma que sitúa el trabajo de cuidados como aspecto determinante de la reproducción social y de las condiciones de vida de la población (Picchio, 2005).

Por una parte, cabe destacar que se hace una crítica epistemológica y metodológica a la teoría neoclásica por el sesgo androcéntrico que implica el "agente representativo", pues atribuye al hombre económico (homo economicus) características que considera universales, pero que, sin embargo, son propias de un ser humano varón, blanco, adulto, sano y de ingresos medios (Picchio, 2005; Rodríguez, 2015). La crisis del COVID-19 pone en evidencia que factores como el sexo, la edad y la raza-etnia sitúan a las personas en condiciones de poder desiguales para hacer frente a la pandemia, 
así como a las crisis que esta conlleva. Las asimetrías de poder entre las personas tienen repercusiones en las probabilidades de exposición al virus, de acceder a servicios de salud de calidad, de contar con un espacio doméstico seguro y libre de violencia, de disponer de ahorros o medios para teletrabajar o realizar educación a distancia, entre otros. Por tanto, los supuestos detrás de las teorías y las políticas económicas deberían trascender las visiones homogeneizadoras de los sujetos para contar con instrumentos adecuados que den respuesta a las múltiples crisis cristalizadas por la pandemia mediante un enfoque que contribuya a cerrar las brechas.

Por otra parte, las herramientas conceptuales y los estudios de género, desarrollo y macroeconomía han permitido determinar las consecuencias de los procesos de globalización y las políticas financieras y fiscales en las desigualdades de género, en particular en contextos de crisis. También desde esta área de producción de conocimiento se han impulsado marcos de análisis para visibilizar los vínculos entre los niveles macro, meso y micro de la economía (Elson, 1994), considerando que hombres y mujeres ocupan posiciones asimétricas de poder como agentes económicos. En diferentes estudios se analizaron las políticas implementadas en países y regiones en desarrollo en las décadas de 1980 y 1990 y se estableció que las mujeres absorbieron en mayor medida los costos de la crisis de la deuda, los programas de ajuste estructural y los recortes de gasto público, mediante la intensificación del trabajo de cuidados y la precarización de sus condiciones de vida (Grown y Sen, 1987; Elson y Cagatay, 2000; Benería, Berik y Floro, 2015). Posteriormente se realizaron diversos análisis sobre los efectos de la crisis financiera de 2007-2008 y las políticas de austeridad (Elson, 2010; Montaño y Milosavljevic, 2010; Esquivel y Rodríguez, 2014; Fukuda-Parr, Heintz y Seguino, 2015; Bohoslavsky, 2018).

Elson (2010) propone un marco para analizar las dimensiones de género de la crisis financiera en los países en desarrollo, tomando las economías como estructuras que se basan en relaciones de género y que comprenden la esfera de las finanzas, la producción y la reproducción social. Presenta los canales de transmisión del norte global, los efectos inmediatos y las respuestas de los gobiernos, las empresas y las personas correspondientes a las tres esferas. Mediante un análisis aplicado en la Argentina, el Ecuador y México, Esquivel y Rodríguez (2014) analizan los efectos de la crisis económica mundial a través de la contracción del comercio, la reducción de la inversión extranjera directa y las remesas y señalan las consecuencias en los niveles de actividad, empleo e ingresos de hombres y mujeres. A partir del análisis de las respuestas de política señalan que las políticas contracíclicas implementadas no incluyen consideraciones para abordar las desigualdades de género en el diseño ni en la evaluación del impacto. El apoyo a los sectores productivos y la inversión pública se ha orientado a impulsar sectores en los que se concentran los hombres, como la construcción y la infraestructura, y no consideran de forma explícita el objetivo de crear oportunidades de empleo para las mujeres. Estos análisis han sido fundamentales para determinar los sesgos de género contenidos en la política macroeconómica de respuesta a las diferentes crisis y cobran relevancia en el contexto actual, en que los países están impulsando paquetes de estímulo fiscal.

La economía feminista crítica ha cuestionado los enfoques ortodoxos y heterodoxos de la economía por su estrecha definición de la disciplina al reducirla a la economía de mercado. "Los distintos enfoques económicos -sean más keynesianos o más neoliberales-, aunque con diferencias entre sí, mantienen sus análisis centrados exclusivamente en la producción, el consumo y la distribución mercantil, sin considerar el trabajo y las diversas actividades que caen fuera de los márgenes del mercado. Como resultado, dichos análisis no solo son parciales, sino que pueden ser erróneos. Si solo se tiene en cuenta y se analiza una parte de la realidad, pero bajo el supuesto de que es la totalidad, nada asegura que los resultados -estadísticos o de políticas por implementar- sean los adecuados" (CEPAL, 2015, pág. 13). La pandemia de COVID-19 ha puesto de relieve uno de los principales aportes de la economía feminista: el trabajo de cuidados que realizan principalmente las mujeres dentro de los hogares es fundamental para la reproducción de la fuerza laboral y el funcionamiento de las economías 
(Picchio, 2001, 2005, 2009)1'. En este contexto se visibilizan las relaciones de producción dentro de los hogares, donde se producen bienes y servicios para satisfacer las necesidades de las personas por medio del trabajo no remunerado. Las medidas de confinamiento, distanciamiento físico y cierre de centros educativos y laborales han intensificado esta carga de trabajo para las mujeres y profundizado la crisis de los cuidados (CEPAL, 2020d; CEPAL/ONU-Mujeres, 2020). Las necesidades educativas, de atención de salud de personas enfermas y la presión para incrementar los estándares de higiene para prevenir el contagio se han concentrado en el ámbito de los hogares. En sociedades marcadas por patrones culturales patriarcales y discriminatorios, las desigualdades de género parecen profundizarse.

Como resultado de la crisis del COVID-19 y las medidas de confinamiento implementadas a escala mundial, se han difuminado los límites entre el ámbito público y el privado y se ha evidenciado aún más la interdependencia entre el trabajo no remunerado y el trabajo para el mercado. Para comprender cabalmente este fenómeno inédito es preciso retomar los diferentes aportes de la economía feminista, que han permitido determinar los conflictos distributivos y las asimetrías de poder de género dentro de los hogares y los diversos tipos de familias. Se han cuestionado las limitaciones de considerar al hogar como unidad de observación y el supuesto del hogar como organización armónica sin conflictos en la que existe una distribución equitativa de los recursos entre los integrantes y se concilian intereses y "preferencias" para maximizar las utilidades².

A partir de la medición del uso del tiempo es posible visibilizar la división sexual del trabajo y, en particular, los aspectos relacionados con la sobrecarga del trabajo de las mujeres, las distintas características de los tipos de tareas que hacen hombres y mujeres, los arreglos de cuidados que se establecen y las tensiones entre los miembros de los hogares para conciliar el trabajo remunerado y no remunerado. Estos elementos son fundamentales para analizar las posibilidades de insertarse en el mercado laboral, realizar teletrabajo y acceder a recursos e ingresos propios. Es pertinente además observar la estructura de los hogares, los arreglos familiares y los patrones culturales patriarcales vigentes.

La pandemia de COVID-19 devela la importancia de los cuidados de las personas y permite cuestionar uno de los principales sesgos androcéntricos de los mercados laborales, que se basa en el modelo del "trabajador ideal". Este modelo parte del supuesto del "individuo" como un sujeto (hombre) sin responsabilidades domésticas y de cuidado y con una producción invisibilizada de bienes y servicios para su bienestar (Marçal, 2016; Picchio, 2001). Es un sujeto con gran disponibilidad de tiempo para el trabajo en el mercado. Esta ilusión se desmorona en el contexto actual, en el que queda de manifiesto la estrecha interdependencia de las condiciones de vida, las demandas de salud y cuidados de las personas y la disponibilidad para el trabajo remunerado.

Uno de los aportes más relevantes para comprender la crisis actual se relaciona con el propósito de la economía feminista de ampliar las fronteras de la disciplina económica, poniendo la sostenibilidad de la vida en el centro del análisis. El objeto central de la economía debería ser la provisión de bienestar y su dinámica se debería orientar a sostener y reproducir la vida de las personas en condiciones de dignidad (Carrasco, 2006 y 2009). La interdependencia entre las personas y la ecodependencia con la naturaleza y los ecosistemas señalada por diferentes autoras (Carrasco y Tello, 2013; Pérez Orozco, 2014) es cada vez más evidente al analizar las causas y los efectos de la pandemia y las múltiples crisis asociadas. Estos principios son clave para reorientar las economías hacia objetivos de sostenibilidad. Desde esta perspectiva más rupturista dentro de la economía feminista, se observa que hay ejes de dominación que no pueden reducirse al conflicto entre capital y trabajo (remunerado). Existe el conflicto

\footnotetext{
1 Como referencia, cabe destacar que Picchio (2001, pág. 1) define el contenido del trabajo de reproducción social no remunerado como "el cuidado del mantenimiento de los espacios y bienes domésticos, así como el cuidado de los cuerpos, la educación, la formación, el mantenimiento de relaciones sociales y el apoyo psicológico a los miembros de la familia".

2 Véase el artículo sobre el aporte de la conceptualización de Amartya Sen sobre conflictos cooperativos para la conceptualización de los modelos de negociación en la unidad doméstica (Benería, 2008).
} 
estructural entre el proceso de acumulación de capital y la sostenibilidad de la vida, que tienen lógicas contrapuestas. Frente a la preeminencia de la acumulación, hay dimensiones de la vida y vidas enteras sobrantes (Pérez Orozco, 2014). En esta coyuntura, el conflicto entre el capital y la vida se expresa en los diferentes contextos y enmarca las decisiones de política que toman los Estados para responder a la crisis del COVID-19. Por tanto, la cuestión distributiva es central para la economía feminista, pero considerando no solo el capital, sino también los recursos, el tiempo y las mujeres en su diversidad como sujetos.

En este marco, se ha desarrollado el concepto de "economía del cuidado", que comprende todo el trabajo que se realiza de forma no remunerada en el seno de los hogares, así como el trabajo de cuidados que se realiza de forma remunerada en el mercado laboral. Este concepto considera los cuidados para la reproducción de la fuerza de trabajo en los hogares y las dinámicas de los cuidados situadas en los mercados y el empleo o en la prestación de servicios públicos, la provisión de infraestructura y la formulación de políticas públicas. Al relacionar la manera en que las sociedades organizan el cuidado de sus miembros con el sistema económico, se vincula el concepto del cuidado con el valor económico que genera (Montaño y Calderón, 2010).

Si bien los efectos de la crisis del COVID-19 en la autonomía económica de las mujeres constituyen un fenómeno en desarrollo, los elementos que se presentan brindan antecedentes de investigación y herramientas conceptuales que permiten comprender las dinámicas de la crisis actual.

\section{Mercado laboral, tiempo e ingresos: la autonomía económica de las mujeres}

En América Latina y el Caribe las desigualdades de género son un rasgo estructural e implican una distribución inequitativa del poder, los recursos, la riqueza y el tiempo. Los nudos estructurales de la desigualdad de género están en la base de la insostenibilidad del estilo de desarrollo dominante y refieren a la desigualdad socioeconómica y la persistencia de la pobreza; los patrones culturales patriarcales discriminatorios y violentos y el predominio de la cultura del privilegio; la rígida división sexual del trabajo y la injusta organización social del cuidado, y la concentración del poder. Estos nudos se refuerzan entre sí y limitan el pleno goce de los derechos de las mujeres y el avance hacia la igualdad sustantiva.

La reducción de la desigualdad de ingresos en la última década, producto de la combinación de crecimiento económico y políticas progresivas, no trajo consigo una distribución más equilibrada del capital y el trabajo (remunerado). Esta asimetría se exacerba para las mujeres, que acceden en menor proporción al capital y se concentran en sectores en los que el trabajo remunerado es más precario. A pesar de las iniciativas para reducir la pobreza, la feminización de la pobreza no disminuyó por el carácter estructural de la desigualdad de género en la región (CEPAL, 2017).

La heterogeneidad estructural de los países de la región se refuerza con la actual división sexual del trabajo y, por lo tanto, es imprescindible no desconocer la interdependencia entre el empleo y el trabajo no remunerado que se realiza en los hogares. Esto se traduce en mercados laborales que presentan un patrón de segregación laboral de género caracterizado por una menor participación de las mujeres en el empleo, su concentración en sectores económicos y ocupaciones de menor productividad, menores niveles de ingresos y bajo acceso a la protección social. Los fenómenos de segregación laboral de género vertical y horizontal, a los que se suman las brechas salariales, persisten en las actuales estructuras productivas poco diversificadas, intensivas en recursos naturales, con bajo contenido tecnológico y con fuertes vulnerabilidades asociadas a las asimetrías internacionales y la restricción externa. También persisten las barreras al acceso de las mujeres a los recursos de producción, como el crédito, la tierra, el agua, la capacitación, las tecnologías y el tiempo (CEPAL, 2019). 
Actualmente las mujeres están siendo afectadas por la disminución de la participación laboral y la intensificación de la demanda de cuidados. Esto supone un retroceso en los avances alcanzados (aunque limitados) en la incorporación de las mujeres a la fuerza laboral. Cabe destacar que, en las últimas décadas, América Latina y el Caribe fue la región del mundo que registró la mayor reducción porcentual en la brecha de género de participación laboral, que se concentró sobre todo entre 1997 y 2007 (7 puntos porcentuales). Esta tendencia fue impulsada por una disminución constante de la tasa de participación masculina, combinada con un aumento en la proporción de mujeres que ingresan a la fuerza de trabajo. Entre 1997 y 2007, la tasa de participación de las mujeres se incrementó 5,3 puntos porcentuales (Vaca Trigo, 2019). Sin embargo, desde entonces ha aumentado levemente, hasta alcanzar un promedio del 51,3\% en 2019, 22,7 puntos porcentuales por debajo de la de los hombres (CEPAL/OIT, 2020).

Desde una perspectiva sectorial, se observa que las mujeres se concentran en sectores como el comercio, el turismo, la industria manufacturera y en sectores de la economía del cuidado (servicio doméstico, salud y enseñanza). El turismo, el comercio y la manufactura son sectores expuestos a los canales de transmisión externos de la crisis, como el cierre total o parcial de las fronteras, la reducción de la demanda de turismo y la interrupción de las cadenas globales de valor. En el contexto de la crisis del COVID-19 son precisamente estos sectores y el servicio doméstico los que sufrieron mayores pérdidas en términos de volumen de la producción y empleo. El 56,9\% del empleo de las mujeres y el 40,6\% del empleo de los hombres en América Latina se concentra en sectores de alto riesgo económico y de pérdida de empleo (CEPAL, 2020g). Por otra parte, si bien las mujeres ocupadas en los sectores de la salud y la enseñanza no vieron amenazados sus puestos de trabajo, fueron quienes tuvieron que responder a la crisis en la primera línea, al tener que hacerse cargo de los cuidados y prevenir la propagación del virus intensificando sus jornadas laborales y exponiéndose al contagio o al tener que dar respuesta a las nuevas demandas de la educación a distancia.

En términos de participación en el mercado laboral, la crisis originada por el COVID-19 presenta características distintas con respecto a las crisis económicas anteriores y, en particular, se observa una considerable salida de la fuerza de trabajo de hombres y mujeres de la región (véase el cuadro 1). En las crisis previas, la contracción de la actividad económica afectó de manera diferenciada a hombres y mujeres, con un incremento de la participación laboral de las mujeres y un mayor efecto de "trabajador desalentado" entre los hombres ${ }^{3}$ (CEPAL, 2014; Espino, 2012; Antonopoulos, 2009; Arroyo y otros, 2010; Montaño y Milosavljevic, 2010). En el caso de las mujeres, por tanto, opera el efecto de "trabajador secundario", referido a quienes entran al mercado laboral en coyunturas de crisis para aumentar las posibilidades de obtener un ingreso para sus hogares en contextos de alto desempleo de los hombres (Esquivel y Rodríguez, 2014).

\footnotetext{
3 Los trabajadores desalentados son aquellas personas que están fuera de la fuerza de trabajo y que no realizaron actividades de búsqueda por motivos vinculados con el mercado laboral (fracaso anterior para encontrar un puesto de trabajo adecuado; falta de experiencia, calificaciones o puestos de trabajo adaptados a las competencias laborales de la persona; escasez de puestos de trabajo en la zona; la persona es considerada demasiado joven o demasiado mayor por los posibles empleadores).
} 


\section{Cuadro 1}

América Latina y el Caribe (14 países): variación acumulada del PIB en crisis económicas previas y en la crisis debida a la pandemia de COVID-19 y variación de los principales indicadores del mercado laboral por sexo

(En porcentajes)

\begin{tabular}{|c|c|c|c|c|c|c|c|c|}
\hline & & \multirow[t]{2}{*}{$\begin{array}{l}\text { Variación del } \\
\text { crecimiento } \\
\text { del PIB }\end{array}$} & \multicolumn{2}{|c|}{$\begin{array}{c}\text { Variación de la tasa } \\
\text { de participación } \\
\text { laboral }\end{array}$} & \multicolumn{2}{|c|}{$\begin{array}{l}\text { Variación de la tasa } \\
\text { de desocupación }\end{array}$} & \multicolumn{2}{|c|}{$\begin{array}{l}\text { Variación de la } \\
\text { tasa de ocupación }\end{array}$} \\
\hline & & & Mujeres & Hombres & Mujeres & Hombres & Mujeres & Hombres \\
\hline \multirow[t]{7}{*}{ Crisis anteriores } & Brasil (entre 1989 y 1990) & $-4,4$ & 1,0 & $-1,1$ & 3,7 & 20,2 & 0,8 & $-1,1$ \\
\hline & $\begin{array}{l}\text { Argentina } \\
\text { (entre } 2000 \text { y 2002) }\end{array}$ & $-14,8$ & 2,5 & $-1,8$ & 9,7 & 30,4 & 0,5 & $-6,5$ \\
\hline & $\begin{array}{l}\text { Uruguay } \\
\text { (entre } 2000 \text { y 2002) }\end{array}$ & $-14,0$ & 0,2 & $-1,9$ & 24,9 & 24,4 & $-4,4$ & $-4,4$ \\
\hline & $\begin{array}{l}\text { Paraguay } \\
\text { (entre } 2008 \text { y 2009) }\end{array}$ & $-4,0$ & 2,5 & $-0,2$ & 7,6 & 26,8 & 1,9 & $-1,5$ \\
\hline & $\begin{array}{l}\text { Venezuela (República } \\
\text { Bolivariana de) } \\
\text { (entre } 2008 \text { y 2009) }\end{array}$ & $-3,2$ & 1,8 & $-0,3$ & 6,0 & 6,3 & 0,8 & $-1,1$ \\
\hline & & \multirow{2}{*}{$\begin{array}{l}\text { Proyección del } \\
\text { crecimiento } \\
\text { del PIB } 2020\end{array}$} & \multicolumn{2}{|c|}{$\begin{array}{c}\text { Variación de la tasa } \\
\text { de participación } \\
\text { laboral }^{\mathrm{a}}\end{array}$} & \multicolumn{2}{|c|}{$\begin{array}{l}\text { Variación de la tasa } \\
\text { de desocupación }{ }^{\mathrm{a}}\end{array}$} & \multicolumn{2}{|c|}{$\begin{array}{l}\text { Variación de la tasa } \\
\text { de ocupación }{ }^{\mathrm{a}}\end{array}$} \\
\hline & & & Mujeres & Hombres & Mujeres & Mujeres & Hombres & Mujeres \\
\hline \multirow[t]{13}{*}{ Crisis del COVID-19 } & Argentina & $-9,1$ & $-8,7$ & $-12,2$ & 2,3 & 2,6 & $-8,7$ & $-12,4$ \\
\hline & $\begin{array}{l}\text { Bolivia (Estado } \\
\text { Plurinacional de) }\end{array}$ & $-5,2$ & $-7,3$ & $-6,9$ & 3,0 & 4,4 & $-8,6$ & $-9,6$ \\
\hline & Brasil & $-9,2$ & $-7,1$ & $-6,2$ & 0,8 & 1,7 & $-6,5$ & $-6,7$ \\
\hline & Chile & $-7,9$ & $-9,0$ & $-7,2$ & 11,7 & 9,4 & $-12,9$ & $-12,8$ \\
\hline & Colombia & $-5,6$ & $-11,4$ & $-10,1$ & 3,9 & 5,7 & $-12,2$ & $-13,0$ \\
\hline & Costa Rica & $-5,5$ & $-6,2$ & $-4,6$ & 15,4 & 10,1 & $-12,1$ & $-11,3$ \\
\hline & Jamaica & $-5,3$ & $-4,3$ & $-3,5$ & 3,8 & 5,7 & $-5,9$ & $-7,1$ \\
\hline & México & $-9,0$ & $-9,6$ & $-16,1$ & $-0,9$ & 1,7 & $-8,9$ & $-16,6$ \\
\hline & Nicaragua & $-8,3$ & $-4,6$ & $-3,3$ & $-0,5$ & 0,4 & $-4,1$ & $-3,4$ \\
\hline & Paraguay & $-2,3$ & $-6,3$ & $-2,5$ & 1,0 & $-0,5$ & $-6,4$ & $-2,0$ \\
\hline & Perú & $-13,0$ & $-28,4$ & $-24,9$ & 3,5 & 6,4 & - & - \\
\hline & República Dominicana & $-5,3$ & $-8,8$ & $-7,9$ & $-5,2$ & $-1,8$ & $-5,7$ & $-6,3$ \\
\hline & Uruguay & $-5,0$ & $-3,6$ & $-3,8$ & 0,6 & 1,2 & $-3,6$ & $-4,3$ \\
\hline
\end{tabular}

Fuente: Comisión Económica para América Latina y el Caribe (CEPAL), "Enfrentar los efectos cada vez mayores del COVID-19 para una reactivación con igualdad: nuevas proyecciones", Informe Especial COVID-19, № 5, Santiago, 2020; Panorama Social de América Latina, 2014 (LC/G.2635-P), Santiago, 2014; Centro de Estudios Distributivos, Laborales y Sociales (CEDLAS); encuestas de hogares y datos oficiales de los respectivos países.

a La variación de los principales indicadores del mercado laboral en el caso de las crisis pasadas corresponde a la variación acumulada para los períodos señalados en CEPAL (2014) y para la crisis del COVID-19 corresponde a la variación interanual de los indicadores para el segundo trimestre 2019/2020, a excepción de Jamaica, donde corresponde a la variación interanual del mes de julio 2019/2020, y México y el Uruguay, donde la variación interanual corresponde al mes de mayo 2019/2020.

En el segundo trimestre de 2020, la proporción de mujeres que se retiraron del mercado laboral fue mayor que la de los hombres. Según las cifras oficiales de los países de la región sobre la variación en el nivel de la ocupación entre el trimestre abril-junio de 2020 y el mismo período del año anterior, la contracción de la ocupación también fue mayor entre las mujeres. En el caso del Perú se observa una reducción de la proporción de mujeres ocupadas del 45\%, mientras en Chile, Colombia y Costa Rica, 1 de cada 4 mujeres salió de la ocupación con respecto al mismo trimestre de 2019 (véase el gráfico 1). 


\section{Gráfico 1}

América Latina (9 países): variación en la población ocupada por sexo, trimestre abril-junio, 2020/2019

(En porcentajes)

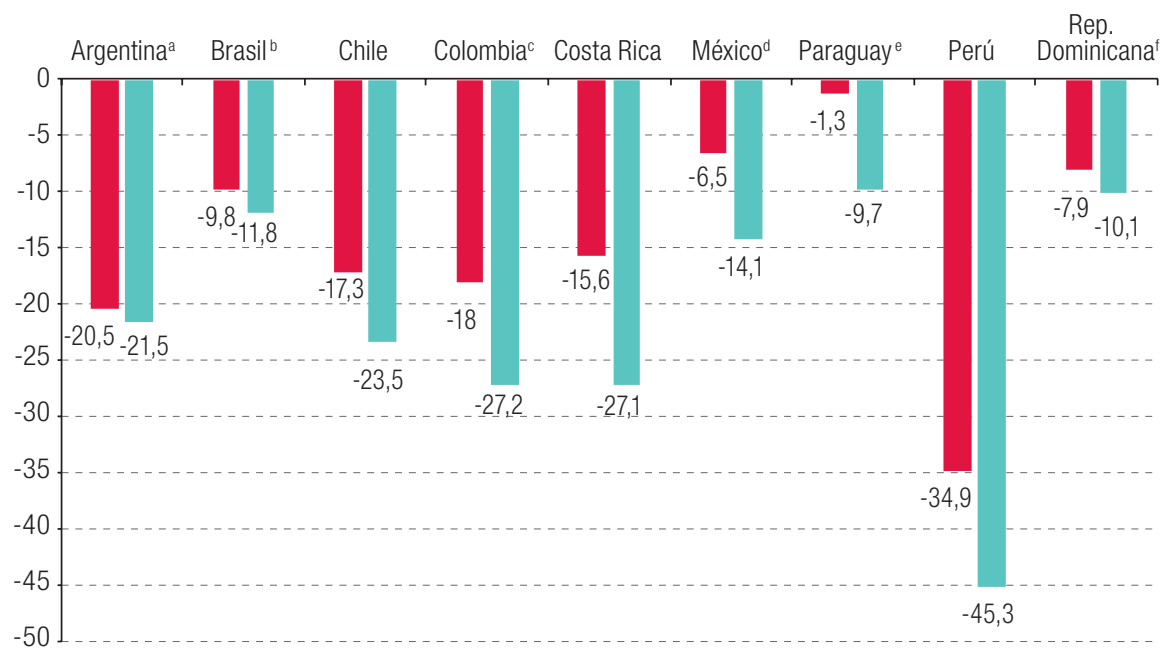

Hombres Mujeres

Fuente: Comisión Económica para América Latina y el Caribe (CEPAL), sobre la base de Instituto Nacional de Estadística y Censos (INDEC), Encuesta Permanente de Hogares (EPH), Buenos Aires, 2020; Instituto Brasileño de Geografía y Estadística (IBGE), Pesquisa Nacional por Amostra de Domicílios Contínua - PNAD Contínua, Brasilia, 2020; Instituto Nacional de Estadística (INE), Boletín estadístico: empleo trimestral, NN261, Santiago, 2020; Departamento Administrativo Nacional de Estadística (DANE), "Boletín técnico: Gran Encuesta Integrada de Hogares (GEIH) junio 2020", Bogotá, 2020; Instituto Nacional de Estadística y Censos (INEC), "Encuesta Continua de Empleo al segundo trimestre de 2020: resultados generales", San José, 2020; Instituto Nacional de Estadística y Geografía (INEGI), Encuesta Nacional de Ocupación y Empleo (ENOE) 2019, Aguascalientes, 2019; Encuesta Nacional de Ocupación y Empleo (Nueva Edición) (ENOEN) 2020, Aguascalientes, 2020; Dirección General de Estadística, Encuestas y Censos (DGEEC), "Boletín trimestral de empleo: EPHC 2do trimestre 2020", Asunción, 2020; Instituto Nacional de Estadística e Informática (INEI), "Comportamiento de los indicadores de mercado laboral a nivel nacional", Informe Técnico, №3, Lima, 2020; Banco Central de la República Dominicana, Encuesta Nacional Continua de Fuerza de Trabajo (ENCFT), Santo Domingo, 2020.

a Segundo trimestre de 2020. Datos para 31 aglomerados urbanos.

b Segundo trimestre de 2020, personas de 14 años de edad o más ocupadas en la semana de referencia, según el sexo.

c El dominio total nacional no incluye la población de los departamentos de Amazonas, Arauca, Casanare, Guainía, Guaviare, Putumayo, Vaupés, Vichada y San Andrés.

d La variación corresponde a julio de 2019-julio de 2020. Los datos para 2020 corresponden a información preliminar.

e No incluye los departamentos de Boquerón y Alto Paraguay.

f Datos trimestrales abril-junio de 2020. Los datos para 2020 corresponden a información preliminar.

En los últimos años, la mayor participación laboral de las mujeres y la desaceleración de la creación de empleos se han traducido en un aumento del desempleo femenino, que sigue siendo superior al de los hombres. A diferencia de lo que suele ocurrir en otras crisis de origen económico, la tasa de desocupación aumentó más para los hombres que para las mujeres. Esto no necesariamente implica que los hombres hayan sido más afectados por la crisis que las mujeres, sino que subraya la necesidad de complementar el análisis de la coyuntura laboral basado en la tasa de desocupación con la revisión de otros indicadores relativos a la participación en la fuerza laboral y la distribución del trabajo no remunerado (CEPAL/OIT, 2020). En la coyuntura actual se observa una disminución de la tasa de participación económica de las mujeres pues, frente a la pérdida del empleo, las circunstancias de la crisis y las altas demandas de cuidados, muchas de ellas no emprendieron la búsqueda de un nuevo empleo. Las últimas proyecciones de la CEPAL indican que habrá una contracción de al menos 6 puntos porcentuales en la tasa de participación laboral de las mujeres a nivel regional (CEPAL, 2020h).

Por otra parte, tanto para los hombres como para las mujeres, la principal fuente de ingresos proviene de la actividad laboral en forma de sueldos y salarios (el 65\% y el 67,2\% respectivamente) (véase el gráfico 2). Esto indica la importancia del salario en la composición de los ingresos de las personas 
en la región y, por ende, el peso del mercado laboral en la autonomía económica y la mitigación de los efectos de las crisis. Además del propio peso de los sueldos y salarios en la composición de los ingresos de las personas, cuando este componente proviene del sector formal del empleo habilita el acceso a diferentes prestaciones sociales y derechos.

\section{Gráfico 2}

América Latina (promedio ponderado de 16 países): composición de los ingresos de las personas de 20 a 59 años según la fuente de los ingresos y la situación con respecto a la pobreza, por sexo, alrededor de 2019

(En porcentajes)

\section{A. Población total}

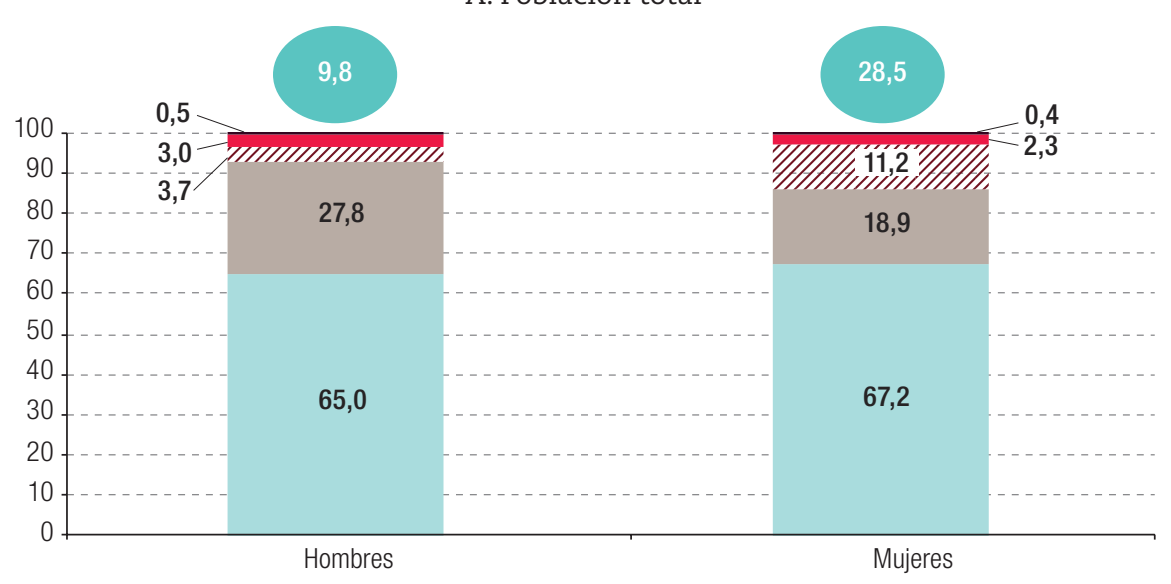

B. Población en situación de pobreza

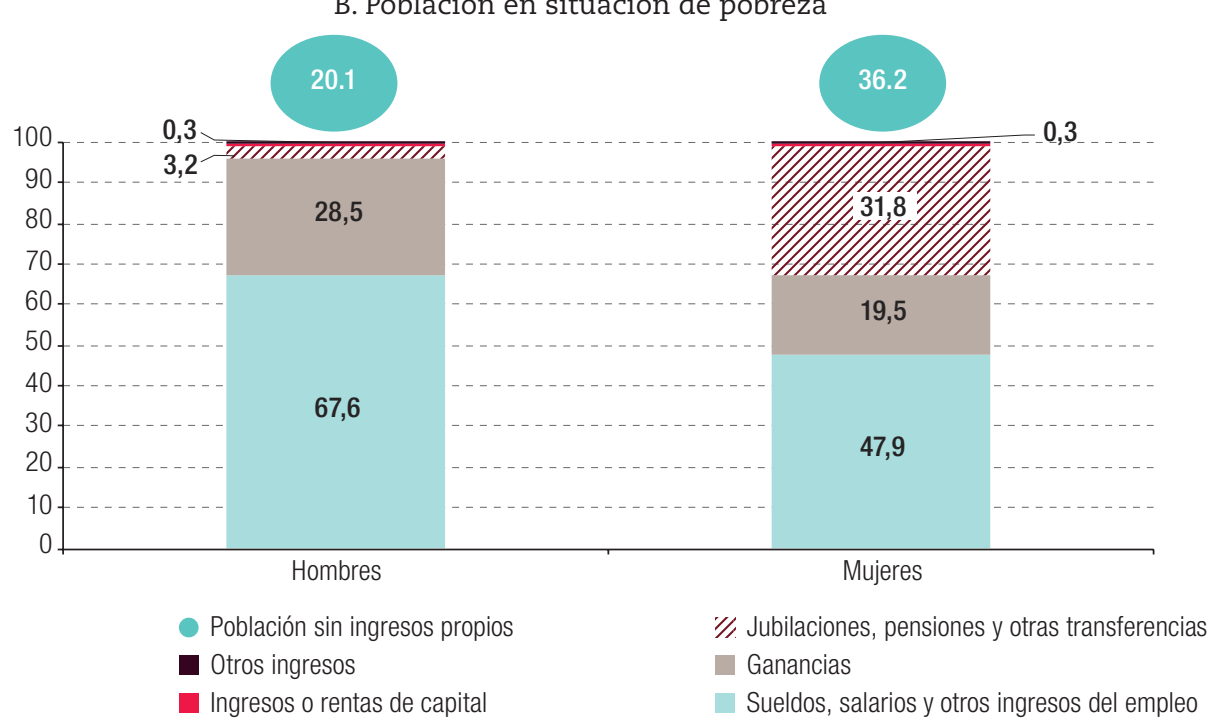

Fuente: Comisión Económica para América Latina y el Caribe (CEPAL), sobre la base de tabulaciones especiales de las encuestas de hogares de los respectivos países.

Nota: Los ingresos de las personas pueden provenir de diversas fuentes: de la inserción en el mercado laboral en forma de sueldos y salarios o como ganancias del autoempleo; de la renta derivada de la propiedad de patrimonio físico o financiero y de transferencias relacionadas con la trayectoria laboral de las personas o con alguna condición por la cual la persona reciba dinero a título personal. Las transferencias pueden provenir del Estado y, a su vez, ser contributivas (como las jubilaciones), no contributivas (como las pensiones por discapacidad o los programas especiales de alivio de la pobreza) o derivar de un vínculo con un familiar o exfamiliar en forma de remesas u obligaciones vinculadas a la disolución conyugal.

Los datos corresponden a 2019 para Argentina, Brasil, Costa Rica, Ecuador, El Salvador, Honduras, Panamá, Paraguay, Perú, República Dominicana y Uruguay; a 2018 para Bolivia (Estado Plurinacional de), Colombia y México; a 2017 para Chile, y a 2014 para Guatemala. 
En el caso de los hombres, el último tercio de la composición de los ingresos corresponde a las ganancias, mientras para las mujeres esta categoría representa un 18,9\% (véase el gráfico 2). En América Latina, este tipo de ingresos no solo está asociado con el ámbito empresarial y el manejo de activos financieros, áreas en que las mujeres tienen menor presencia, sino también con las ganancias del trabajo por cuenta propia o independiente, una modalidad muy extendida en la región (trabajo formal e informal). La presencia de las mujeres en esta última categoría es muy importante. En este caso, las ganancias de las mujeres pueden ser más inestables y presentar mayores niveles de desprotección del empleo ya que están fuertemente asociadas al trabajo informal (CEPAL, 2016).

Cabe destacar que el Observatorio de Igualad de Género de América Latina y el Caribe (OIG) releva el indicador de personas sin ingresos propios como un aspecto central del análisis de la autonomía de las mujeres y una alternativa para analizar los recursos individuales sin considerar al hogar como unidad de análisis ni asumir una distribución equitativa de los recursos entre todos los miembros del hogar (CEPAL, 2020e). Se observa que 1 de cada 3 mujeres de 20 a 59 años que viven en hogares pobres (36\%) no tiene ningún ingreso propio, en contraste con la situación de los hombres con iguales características, para quienes la relación es de 1 de cada 5 (véase el gráfico 2B).

Entre las personas que sí cuentan con ingresos propios se observan marcadas diferencias en la composición de los ingresos de hombres y mujeres. El caso más agudo se observa con respecto a la participación de las transferencias del Estado. La distinción de esta diferencia en la fuente de los ingresos es muy importante por su fuerte vínculo con la formulación de políticas públicas de mitigación de la crisis actual. Las transferencias constituyen el 11,2\% de los ingresos de las mujeres y representan solamente el 3,7\% de los ingresos de los hombres (véase el gráfico 2).

El análisis de las desigualdades de género según la situación con respecto a la pobreza en los hogares revela que las transferencias representan el 31,8\% de los ingresos de las mujeres que viven en hogares pobres, mientras entre los hombres esta categoría alcanza solamente el 3,2\% (véase el gráfico 2).

Cabe destacar que, si bien más mujeres que hombres reciben transferencias de programas para poner fin a la pobreza o mitigar los efectos de las crisis económicas, estas no son un ingreso del que puedan disponer libremente pues, en el marco de los programas de transferencias condicionadas, las mujeres suelen cumplir el papel de "administradoras" de los recursos para el hogar. Las mujeres no acceden a estos recursos como sujetos de derecho propio, sino como beneficiarias operativas. Esto refuerza su papel de mujeres-madres y principales responsables del trabajo de cuidado. Los efectos de los programas de transferencias condicionadas en las mujeres dependen de factores como el monto de la transferencia, la cobertura y las condicionalidades asociadas. No obstante, se ha recomendado revisar el enfoque de estos programas que refuerzan el maternalismo social de las políticas e incluso evaluar la supresión de las condicionalidades y fomentar el principio de corresponsabilidad (CEPAL, 2016).

La generalización de estos instrumentos de política social sin una adecuada planificación ni escenarios de reconstrucción sostenibles y con igualdad plantea el riesgo de alejar a las mujeres del empleo de calidad y las políticas universales de seguridad social (Montaño y Milosavljevic, 2010). En la coyuntura actual, en la que diferentes países de la región han reforzado sus programas de transferencias, esta noción adquiere relevancia como orientación para evitar la reproducción de sesgos de género en la política pública. Por otra parte, los criterios para terminar con este tipo de programas generalmente responden a metas e indicadores macroeconómicos que no necesariamente tienen en cuenta las dificultades de las mujeres para acceder a un nuevo ingreso propio luego de finalizada la prestación.

La crisis sanitaria y económica provocada por la enfermedad por coronavirus (COVID-19) profundiza la lógica de la asignación focalizada de recursos monetarios sin un cuestionamiento de los efectos perpetuadores de la desigualdad de género que estos programas pueden tener si no se analizan a la luz de los impactos diferenciados de género. Si bien estas son medidas imprescindibles 
a corto plazo para amortiguar la reducción de los ingresos de los hogares y mitigar el aumento de la pobreza, es crucial que su diseño integre medidas adicionales a mediano y largo plazo ya que, de ser esta la única solución, se perpetuarían mecanismos que pueden redundar en la profundización de las brechas de género.

\section{Reflexiones para una recuperación transformadora}

La crisis causada por la pandemia de COVID-19 ha puesto en evidencia la estrecha interdependencia entre los procesos económicos orientados al mercado y los procesos de reproducción social. Ante la falta de políticas de redistribución social de los cuidados y la persistencia de patrones culturales patriarcales que se traducen en expectativas diferenciadas por género, las mujeres están absorbiendo la sobrecarga de cuidados y, en consecuencia, aumentan los obstáculos para su participación en el mercado laboral.

Esta crisis está afectando los niveles de actividad, empleo e ingresos de las mujeres y precarizando sus condiciones de vida. Se observa un deterioro de los indicadores asociados al vínculo de las mujeres con el mercado laboral, en particular una reducción de la participación laboral y una concentración de las mujeres en sectores expuestos a la pérdida de empleo y de ingresos. Por tanto, se está profundizando la división sexual del trabajo y poniendo en riesgo los escasos avances en términos de autonomía económica de las mujeres alcanzados en los años previos a la crisis.

Los Gobiernos de la región han impulsado diferentes iniciativas para hacer frente a las dimensiones de género de la pandemia y sus efectos socioeconómicos (CEPAL, 2020b). Las medidas se han concentrado principalmente en abordar la violencia de género contra las mujeres, la protección del empleo y los ingresos, el acceso a prestaciones y transferencias (ampliando cobertura y montos) y algunos aspectos de la economía del cuidado. Hasta el momento, las acciones parecen focalizarse en medidas de contención de los efectos a corto plazo, sin capacidad para transformar la desigualdad a mediano y largo plazo. En menor medida se ha contemplado la incorporación de la perspectiva de género en los paquetes de estímulo fiscal orientados a la reactivación a mediano y largo plazo, que generaría fuentes de ingresos más estables para las mujeres e impulsaría el logro de la autonomía económica.

En algunas ocasiones, el enfoque de las políticas reproduce los sesgos de género al considerar a los hombres como principales proveedores de los hogares y a las mujeres como trabajadoras con un ingreso secundario o principales responsables de los cuidados. Se impulsan medidas para mantener el poder adquisitivo de los hogares, sin visibilizar las dinámicas dentro del hogar y bajo el supuesto de una distribución equitativa de los recursos entre sus integrantes.

Por otra parte, las medidas específicas que consideran a las mujeres como población vulnerable o como grupo homogéneo se enmarcan en una visión que perpetúa las asimetrías de género y no modifican las causas estructurales de la desigualdad. El enfoque de derechos es crucial para reconocer a las mujeres como sujetos con capacidad de tomar decisiones y a los Estados como responsables de garantizar sus derechos. Además, los factores como la edad, la raza-etnia y los ingresos son necesarios para determinar la exposición de las mujeres y su capacidad de respuesta a los diferentes efectos socioeconómicos de la crisis. Por lo dicho, se requiere impulsar el diseño de políticas de corto, mediano y largo plazo con un enfoque interseccional, que reconozcan la diversidad de las mujeres y avancen hacia la igualdad sustantiva.

La crisis del COVID-19 debe ser una oportunidad para impulsar una nueva trayectoria en el estilo de desarrollo en la región, que contribuya a superar los nudos estructurales de la desigualdad de género y avanzar hacia el logro de la autonomía de las mujeres. 
Con este horizonte, la incorporación de la perspectiva de género en las políticas macroeconómicas, productivas y sociales de reactivación es clave para transformar las economías. En este sentido, no solo se requiere incorporar plenamente a las mujeres en sectores estratégicos y dinamizadores de la reactivación, sino también hacer cambios profundos en el enfoque de las políticas, la distribución del poder y del tiempo, la asignación de los recursos y los incentivos económicos, basando la respuesta política en los principios de justicia de género, sostenibilidad e igualdad ${ }^{4}$. Esto implica repensar los patrones de producción, consumo y distribución sostenible y reorientar las finanzas hacia la economía real. También supone avanzar en el proceso de diversificación productiva en sectores estratégicos para la sostenibilidad de la vida, que contribuyan a la generación de empleo de calidad, a superar la segregación laboral de género y a la redistribución social de los cuidados desde el enfoque de la corresponsabilidad.

Un entorno macroeconómico propicio para una reactivación con igualdad es imprescindible. Se debe respetar el espacio de políticas de los Estados para que puedan hacer uso de instrumentos frente a los choques de la crisis desde un enfoque que garantice los derechos de las mujeres y promueva el cierre de las brechas estructurales. Si bien el espacio fiscal de los países es limitado, es posible ampliarlo a través del aumento de la progresividad de los sistemas tributarios, mediante impuestos directos a la renta y la riqueza, combatiendo la evasión y la elusión fiscal y los flujos financieros ilícitos (CEPAL, 2019 y 2020a). La movilización de recursos públicos adicionales es necesaria para reforzar el financiamiento de las políticas de igualdad de género en áreas estratégicas para las mujeres como los servicios de cuidados, de salud sexual y reproductiva y de atención a situaciones de violencia por razones de género. Las propuestas de austeridad fiscal y de políticas procíclicas basadas en el supuesto de que el mercado es el mecanismo de asignación de recursos más eficiente no conducirían a cerrar las brechas de desigualdad. Por tanto, se recomienda impulsar políticas fiscales contracíclicas que integren en su diseño la perspectiva de género, a fin de revertir los efectos de la crisis en la autonomía de las mujeres y promover una reactivación sostenible y transformadora que permita alcanzar la igualdad sustantiva.

\section{Bibliografía}

Antonopoulos, R. (2009), "The current economic and financial crisis: a gender perspective", Working Paper, № 562, Annandale-On-Hudson, The Levy Economics Institute of Bard College, mayo.

Arroyo, L. y otros (2010), "Los efectos de la crisis sobre las mujeres: empleo, segregación ocupacional y modelo productivo", Informes de la Fundación, № 17, Madrid, Fundación $1^{\circ}$ de Mayo, marzo.

Benería, L. (2008), "De la 'armonía' a los 'conflictos cooperativos': la contribución de Amartya Sen a la teoría de la unidad doméstica", Araucaria, vol. 10, № 20, Sevilla, Universidad de Sevilla, octubre.

Benería, L., G. Berik y M. Floro (2015), Gender, Development and Globalization: Economics as if All People Mattered, Abingdon, Routledge.

Bohoslavsky, J. (2018), Informe del Experto Independiente sobre las consecuencias de la deuda externa y las obligaciones financieras internacionales conexas de los Estados para el pleno goce de todos los derechos humanos, sobre todo los derechos económicos, sociales y culturales: impacto de las reformas económicas y medidas de austeridad en los derechos humanos de las mujeres (A/73/179), Nueva York, Naciones Unidas, julio.

Carrasco, C. (2009), "Mujeres, sostenibilidad y deuda social", Revista de Educación, Madrid, Ministerio de Educación, Cultura y Deporte.

(2006), "La economía feminista: una apuesta por otra economía", Estudios sobre género y economía, M. Vara (ed.), Madrid, Akal.

\footnotetext{
${ }^{4}$ Entre otros sectores estratégicos, en CEPAL (2020a) se hace referencia a la economía digital, la industria manufacturera de la salud y el turismo sostenible.
} 
Carrasco, C. y E. Tello (2013), "Apuntes para una vida sostenible", Tejiendo alianzas para una vida sostenible: consumo crítico, feminismo y soberanía alimentaria, X. Montagut, C. Murias y L. Vega (coords.), Barcelona, Xarxa de Consum Solidari/Marcha Mundial de las Mujeres.

CEPAL (Comisión Económica para América Latina y el Caribe) (2020a), Construir un nuevo futuro: Una recuperación transformadora con igualdad y sostenibilidad (LC/SES.38/3-P/Rev.1), Santiago, octubre. (2020b), "Medidas y acciones a nivel nacional", Santiago, Observatorio COVID-19 en América Latina y el Caribe [en línea] https://www.cepal.org/es/temas/covid-19.

(2020c), "Enfrentar los efectos cada vez mayores del COVID-19 para una reactivación con igualdad: nuevas proyecciones", Informe Especial COVID-19, № 5, Santiago, julio.

(2020d), "La pandemia del COVID-19 profundiza la crisis de los cuidados en América Latina y el Caribe", Informe COVID-19, Santiago, abril.

(2020e), "Población sin ingresos propios por sexo", Santiago, Observatorio de Igualdad de Género de América Latina y el Caribe (OIG) [en línea] http://oig.cepal.org/es/indicadores/poblacion-sin-ingresospropios-sexo.

(2020f), "América Latina y el Caribe ante la pandemia del COVID-19: efectos económicos y sociales", Informe Especial COVID-19, № 1, Santiago, abril.

- (2020g), "La autonomía económica de las mujeres en la recuperación sostenible y con igualdad", Informe Especial COVID-19, Santiago, inédito.

(2020h), Balance Preliminar de las Economías de América Latina y el Caribe 2020 (LC/PUB. 2020/17-P), Santiago.

(2019), La autonomía de las mujeres en escenarios económicos cambiantes (LC/CRM.14/3), Santiago, diciembre.

(2017), Estrategia de Montevideo para la Implementación de la Agenda Regional de Género en el Marco del Desarrollo Sostenible hacia 2030 (LC/CRM.13/5), Santiago, marzo.

(2016), Autonomía de las mujeres e igualdad en la agenda de desarrollo sostenible (LC/G.2686/Rev.1), Santiago, diciembre.

(2015), Informe regional sobre el examen y la evaluación de la Declaración y la Plataforma de Acción de Beijing y el documento final del vigesimotercer período extraordinario de sesiones de la Asamblea General (2000) en los países de América Latina y el Caribe (LC/L.3951), Santiago, febrero. (2014), Panorama Social de América Latina, 2014 (LC/G.2635-P), Santiago, diciembre.

CEPAL/OIT (Comisión Económica para América Latina y el Caribe/Organización Internacional del Trabajo) (2020), "La dinámica laboral en una crisis de características inéditas: desafíos de política", Coyuntura Laboral en América Latina y el Caribe, № 23 (LC/TS.2020/128), Santiago, noviembre.

CEPAL/ONU-Mujeres (Comisión Económica para América Latina y el Caribe/Entidad de las Naciones Unidas para la Igualdad de Género y el Empoderamiento de las Mujeres) (2020), Cuidados en América Latina y el Caribe en tiempos de COVID-19: hacia sistemas integrales para fortalecer la respuesta y la recuperación, Santiago, agosto.

Elson, D. (2010), "Gender and the global economic crisis in developing countries: a framework for analysis", Gender \& Development, vol. 18, № 2, Abingdon, Taylor and Francis, julio. (1994), "Micro, meso, macro: gender and economic analysis in the context of policy reform", The Strategic Silence. Gender and Economic Policy, I. Bakker (ed.), Londres, Zed Press/North-South Institute.

Elson, D. y N. Cagatay (2000), "The social content of macroeconomic policies", World Development, vol. 28, № 7, Amsterdam, Elsevier, julio.

Espino, A. (2012), "La dimensión de género de la crisis ¿Existe una agenda feminista?”, Nueva Sociedad, № 237, Buenos Aires, enero-febrero.

Esquivel, V. y C. Rodríguez (2014), "Addressing the global economic crisis in Mexico, Ecuador and Argentina: implications for gender equality", Gender Perspectives and Gender Impacts of the Global Economic Crisis, R. Antonopoulos (ed.), Nueva York, Routledge.

Fukuda-Parr, S., J. Heintz y S. Seguino (eds.) (2015), Critical and Feminist Perspectives on Financial and Economic Crises, Londres, Routledge, abril.

Grown, C. y G. Sen (1987), Development, Crises and Alternative Visions: Third World Perspectives, Nueva York, Monthly Review Press, diciembre.

Marçal, K. (2016), ¿Quién le hacía la cena a Adam Smith? Una historia de las mujeres y la economía, Barcelona, Random House Mondadori, agosto.

Montaño, S. y C. Calderón (coords.) (2010), El cuidado en acción: entre el derecho y el trabajo, Cuadernos de la CEPAL, № 94 (LC7G.2454-P), Santiago, Comisión Económica para América Latina y el Caribe (CEPAL), septiembre. 
Montaño, S. y V. Milosavljevic (2010), "La crisis económica y financiera: su impacto sobre la pobreza, el trabajo y el tiempo de las mujeres", serie Mujer y Desarrollo, № 98 (LC/L.3168-P), Santiago, Comisión Económica para América Latina y el Caribe (CEPAL), febrero.

Naciones Unidas (2020), Informe: el impacto del COVID-19 en América Latina y el Caribe, Nueva York, julio.

Pérez Orozco, A. (2014), Subversión feminista de la economía: aportes para un debate sobre el conflicto capital-vida, Madrid, Traficantes de Sueños, mayo.

Picchio, A. (2009), "Condiciones de vida: perspectivas, análisis económico y políticas públicas", Revista de Economía Crítica, № 7, Valladolid, Asociación de Economía Crítica (AEC).

(2005), "La economía política y la investigación sobre las condiciones de vida", Por una economía sobre la vida: aportaciones desde un enfoque feminista, G. Cairó y M. Mayordomo (coords.), Barcelona, Icaria Editorial.

(2001), “Un enfoque macroeconómico ‘ampliado' de las condiciones de vida”, Tiempos, trabajo y género, Barcelona, Universidad de Barcelona.

Rodríguez, C. (2015), "Economía feminista y economía del cuidado: aportes conceptuales para el estudio de la desigualdad", Nueva Sociedad, № 256, Buenos Aires, marzo-abril.

Vaca Trigo, I. (2019), "Oportunidades y desafíos para la autonomía de las mujeres en el futuro escenario del trabajo", serie Asuntos de Género, № 154 (LC/TS.2019/3), Santiago, Comisión Económica para América Latina y el Caribe (CEPAL), enero. 